\title{
Genomes of sequence type 121 Listeria monocytogenes strains harbor highly conserved plasmids and prophages
}

\author{
Stephan Schmitz-Esser*, Anneliese Müller, Beatrix Stessl and Martin Wagner \\ Department for Farm Animals and Veterinary Public Health, Institute for Milk Hygiene, University of Veterinary Medicine \\ Vienna, Vienna, Austria
}

OPEN ACCESS

Edited by:

Danilo Ercolini,

Università degli Studi di Napoli

Federico II, Italy

Reviewed by:

Kalliopi Rantsiou,

University of Turin, Italy

David Rodriguez-Lazaro,

Instituto Tecnológico Agrario de

Castilla y León, Spain

Henk Cornelis Den Bakker,

Texas Tech University, USA

${ }^{*}$ Correspondence:

Stephan Schmitz-Esser,

Department for Farm Animals and Veterinary Public Health, Institute for Milk Hygiene, University of Veterinary Medicine Vienna, Veterinaerplatz 1 ,

1210 Vienna, Austria stephan.schmitz-esser@ vetmeduni.ac.at

Specialty section: This article was submitted to Food Microbiology,

a section of the journal

Frontiers in Microbiology

Received: 16 December 2014

Accepted: 13 April 2015

Published: 28 April 2015

Citation:

Schmitz-Esser S, Müller A, Stess/ B and Wagner M (2015) Genomes of

sequence type 121 Listeria

monocytogenes strains harbor highly conserved plasmids and prophages.

Front. Microbiol. 6:380.

doi: 10.3389/fmicb.2015.00380
The food-borne pathogen Listeria (L.) monocytogenes is often found in food production environments. Thus, controlling the occurrence of $L$. monocytogenes in food production is a great challenge for food safety. Among a great diversity of $L$. monocytogenes strains from food production, particularly strains belonging to sequence type (ST)121 are prevalent. The molecular reasons for the abundance of ST121 strains are however currently unknown. We therefore determined the genome sequences of three L. monocytogenes ST121 strains: 6179 and 4423, which persisted for up to 8 years in food production plants in Ireland and Austria, and of the strain 3253 and compared them with available L. monocytogenes ST121 genomes. Our results show that the ST121 genomes are highly similar to each other and show a tremendously high degree of conservation among some of their prophages and particularly among their plasmids. This remarkably high level of conservation among prophages and plasmids suggests that strong selective pressure is acting on them. We thus hypothesize that plasmids and prophages are providing important adaptations for survival in food production environments. In addition, the ST121 genomes share common adaptations which might be related to their persistence in food production environments such as the presence of Tn6188, a transposon responsible for increased tolerance against quaternary ammonium compounds, a yet undescribed insertion harboring recombination hotspot $(\mathrm{RHS})$ repeat proteins, which are most likely involved in competition against other bacteria, and presence of homologs of the L. innocua genes lin0464 and lin0465.

Keywords: Listeria monocytogenes, sequence type 121, persistence, genome, plasmid, prophage

\section{Introduction}

The facultative intracellular pathogen Listeria monocytogenes is responsible for listeriosis, a rare but severe disease in humans and animals, which is acquired primarily through the consumption of contaminated food; particularly "ready-to-eat food" is of high risk (Allerberger and Wagner, 2010; Eurosurveillance Editorial, 2012). L. monocytogenes can survive and grow in multiple natural and man-made habitats, such as soil, marine and fresh water, vegetation, sewage, food processing plants, farm environments, domestic and wild animals (Sauders and Wiedmann, 2007; Ferreira et al., 2014); therefore, controlling L. monocytogenes in food processing environments is a considerable challenge. Long-term survival-also called persistence-for months or even years of various 
L. monocytogenes strains in food production environments has been described by many studies, for reviews see: (Carpentier and Cerf, 2011; Ferreira et al., 2014; Larsen et al., 2014). Persistence describes the repeated occurrence of genetically indistinguishable (determined e.g., by pulsed-field gel electrophoresis or ribotyping) L. monocytogenes strains in the same food production plant over a long time period. However, only relatively few studies have focused on the molecular mechanisms of persistence (Carpentier and Cerf, 2011; Ferreira et al., 2014). So far, only two studies have analyzed genome sequences of persistent L. monocytogenes strains: one study described the genome of L. monocytogenes strain J2818 [sequence type (ST) 11 , serovar $1 / 2 \mathrm{a}$ ], which persisted for 12 years in a food processing plant in the US (Orsi et al., 2008). Another more recent study determined the genome sequences of two persistent ST121 (serovar 1/2a) L. monocytogenes strains isolated from two different fish processing plants in Denmark (Holch et al., 2013). These two studies revealed that prophage diversification is an important driver of L. monocytogenes evolution and suggested that specific genetic determinants may enable longterm persistence in food processing environments. Two models explaining persistence have been proposed (Ferreira et al., 2014): According to the first model, certain L. monocytogenes strains have unique phenotypic and genotypic characteristics facilitating long-term survival in food processing environments. The other model states that persistence is largely a random process and that most L. monocytogenes strains can establish persistence if present in an appropriate niche at an appropriate time (Ferreira et al., 2014). Particularly L. monocytogenes strains of ST121 are often found in food production environments (Ragon et al., 2008; Parisi et al., 2010; Chenal-Francisque et al., 2011; Hein et al., 2011; Holch et al., 2013; Kastbjerg et al., 2014; Martin et al., 2014; Stessl et al., 2014; Wang et al., 2015). However, the molecular mechanisms underlying the phenomenon of persistence are currently unknown. As persistent L. monocytogenes strains in food processing environments greatly increase the risk of (re)contamination of food products and therefore represent a big challenge for food safety, we analyzed the genome sequences of three ST121 L. monocytogenes isolates and compared them with available L. monocytogenes ST121 genomes to identify common and unique genetic traits among ST121 genomes with a particular focus on persistence.

\section{Materials and Methods}

\section{Bacterial Strains Used for Genome Sequencing}

The following three L. monocytogenes strains were potentially persisting in three food processing facilities during several years (Table 1). The strains were assigned to genetic lineage II, serovar 1/2a and ST121. L. monocytogenes 4423 was isolated primarily from product-associated and cheese samples in 2004 from an Austrian cheese processing plant producing semi-hard and hard cheese (Stessl et al., 2014). L. monocytogenes 4423 corresponds to AuB1 and 6179 corresponds to IrlA1 in Stessl et al. (2014). L. monocytogenes 6179 originated from an Irish farmhouse-cheesemaking plant and was mainly isolated from cheeses (Fox et al., 2011a,b; Stessl et al., 2014). L. monocytogenes 3253 was isolated from Austrian deli-meat products and the corresponding food processing environment (FPE). Details on strains are given in Table 1.

\section{DNA Isolation, Genome Sequencing and Genome Analyses}

L. monocytogenes strains were cultivated under aerobic conditions at $37^{\circ} \mathrm{C}$ in brain heart infusion broth (BHI, Merck; with $125 \mathrm{rpm}$ shaking), harvested by centrifugation, the resulting pellet was used for DNA isolation using the QIAGEN genomic-tip columns and buffers according to the recommendations of the manufacturer. For 6179 and 4423 genome sequencing was performed using an Illumina GAII genome analyzer available at the University of Veterinary Medicine Vienna. Sequencing was performed using paired-end sequencing technology and $100 \mathrm{bp}$ read-length using Illumina standard protocols. For 3253 genome sequencing was performed with Illumina MiSeq sequencing technology using 300 bp read length and paired-end sequencing (Microsynth, Balgach, Switzerland). Four (4423) and three (6179 and 3253) million reads were used for a de novo assembly using SeqManNGen (DNASTAR). The average coverage was $145 \times$ for L. monocytogenes 4423, $98 \times$ for L. monocytogenes 6179 , and $205 \times$ for 3253 . This assembly resulted in 35 contigs with a size $>500$ bp for 4423,32 contigs for 6179 , and 12 contigs for 3253 . The contigs were aligned to the L. monocytogenes EGDe genome using the "move contigs" option in MAUVE (Darling et al., 2010) and used for initial genome analyses. PCR and Sanger sequencing was performed to close remaining gaps-this resulted in one contig for 6179 and 12 contigs for 4423. Automatic genome analysis and annotation of the genomes was done using the RAST server (http://rast.nmpdr. org/) (Aziz et al., 2008; Overbeek et al., 2014) and the MicroScope platform (https://www.genoscope.cns.fr/agc/microscope/home/) (Vallenet et al., 2013). Genome comparisons and determination of homologous proteins were done with BlastP, BlastN, and tBlastN (Camacho et al., 2009). Similar to a previous study (Kuenne et al., 2013) we used a similarity cut-off of $60 \%$ amino acid identity and $80 \%$ coverage for identification of homologous proteins. Alignments of genomes, prophages and plasmids were done with MAUVE (Darling et al., 2010). Multilocus sequence typing (MLST) of the sequenced strains was performed with the MLST tool available on the Center for Genomic Epidemiology website [https://cge.cbs.dtu.dk/services/MLST/ (Larsen et al., 2012)]. For comparison other currently available ST121 genomes: the genome sequences of LM_1880 (GenBank accession number AZIZ00000000), a strain isolated from cheese in Italy-for which no information regarding possible persistence is currently available-(Chiara et al., 2014) and N53-1 (GenBank accession number AXDU01000000), a persistent L. monocytogenes strain isolated from a fish production plant in Denmark (Holch et al., 2013; Kastbjerg et al., 2014) and S2_2, S2_3, S10_1, and S10_3 (GenBank accession numbers: JWHJ01000000, JWHK01000000; JWHG01000000, and JWHH01000000), isolated from pork industry in Spain (Ortiz et al., 2010, 2014; Lopez-Alonso et al., 2015) were downloaded from GenBank and loaded onto the RAST server. In the study by Holch and coworkers (Holch et al., 2013) it was stated that the strains La111 and N53-1 were almost identical (no SNPs present), we therefore used only N53-1 for our 


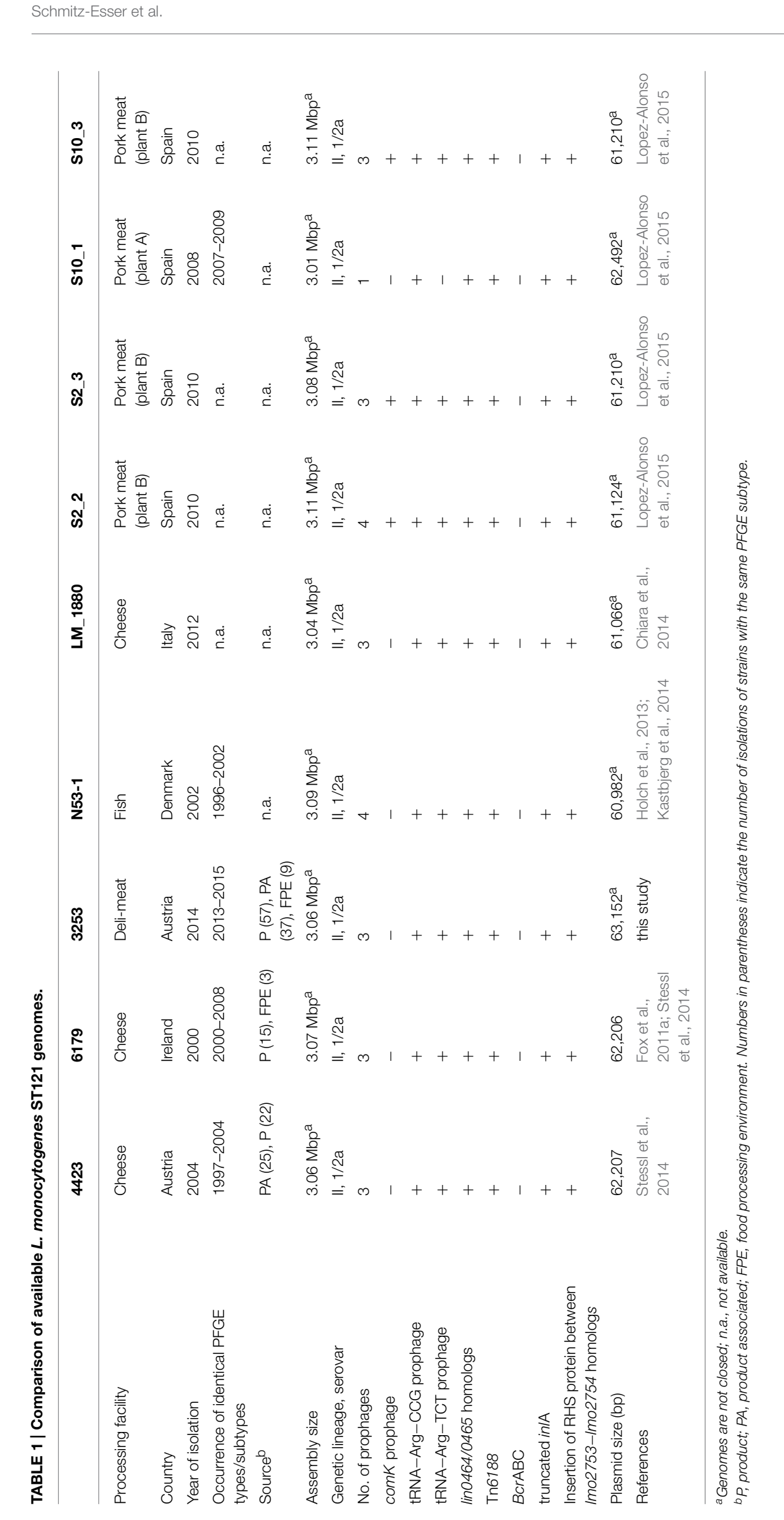

Listeria sequence type 121 genomes 
comparisons. CRISPR regions were analyzed with CRISPRFinder [http://crispr.u-psud.fr/Server/CRISPRfinder.php (Grissa et al., 2007)]. Phylogenetic analyses of plasmid replication initiation protein amino acid sequences was performed with MEGA 6.0 using maximum likelihood based phylogenetic inference and the JTT amino acid substitution model with 1000x bootstrapping (Tamura et al., 2013).

\section{Accession Numbers}

The genome and plasmid sequences have been deposited in the EMBL European nucleotide archive under accession numbers HG813249 and HG813250 for L. monocytogenes 6179 and CBXR010000001 to CBXR010000012 for L. monocytogenes 4423. The L. monocytogenes 3253 whole genome shotgun project has been deposited at DDBJ/EMBL/GenBank under the accession JYJO00000000. The version described in this paper is version JYJO01000000.

\section{Results and Discussion}

Recently, two L. monocytogenes sequence type (ST) 121 strains (4423 and 6179) were found to be potentially persistent in European cheese processing facilities (Fox et al., 2011a,b; Stessl et al., 2014). The genetically highly similar (but not identical) L. monocytogenes strains 4423 and 6179 were recurrently isolated during a timeframe of seven and 8 years (Table 1). Interestingly, a high abundance of L. monocytogenes ST121 in food and persistence in FPE was also observed by other authors (Parisi et al., 2010; Chenal-Francisque et al., 2011; Holch et al., 2013; Althaus et al., 2014; Martin et al., 2014; Ortiz et al., 2014; Wang et al., 2015). Currently, 69 L. monocytogenes ST121 are available in the Institute Pasteur MLST database with a considerable increased isolation history from 2009 to 2014 (http://www.pasteur.fr/recherche/genopole/ PF8/mlst/Lmono.html; accessed on: 19.02.2015).

To get more insight into the genetic features involved in L. monocytogenes ST121 persistence in FPE, the three ST121 strains 4423, 6179, and 3253 were selected for whole genome sequencing and compared with available L. monocytogenes ST121 genomes $(n=6)$.

Genome sequencing followed by gap closing using PCR resulted in one contig for L. monocytogenes 6179 (Table 2), however we were not able to close the genome. The remaining gap probably comprised two consecutive rRNA operons as in the homologous region in L. monocytogenes EGDe. For L. monocytogenes 4423 and 3253, 12 contigs remained (Table 2). The genomes display typical features of $L$. monocytogenes genomes such as assembly sizes between 3.00 and 3.01 Mbp and a genomic $\mathrm{G}+\mathrm{C}$ content of $37.9 \%$, which are in the range found for most Listeria genomes (den Bakker et al., 2010, 2013; Kuenne et al., 2013). In addition, all strains harbor a plasmid (pLM6179, pLM4423, and pLM3253) with sizes of 62.2 (6179 and 4423) and $63.1 \mathrm{kbp}$ (3253) with G+C content of $36.5 \%$. Details on the genomes and plasmids are shown in Table 2.

Overall, all the sequenced ST121 genomes are highly similar to each other and also highly similar to the L. monocytogenes EGDe genome (Figure 1, Table 1); however, one $358 \mathrm{kbp}$ inversion in the 6179 genome between the EGDe homologs Imo1240 (LM6179_1548) and lmo1241 (LM6179_1975) representing homologs of the EDGe genes lmo2589 to lmo2366 including also all three 6179 prophages, was found. This rearrangement most likely explains the slightly different PFGE patterns of 4423 and 6179 reported recently (Stessl et al., 2014). The average nucleotide and amino acid identity between the nine ST121 strains is 99.8 and $99.9 \%$, respectively.

The ST121 genomes harbor between one and four prophages (Table 1). In 6179, 4423, and 3253, one prophage is inserted downstream of the tRNA Arg-TCT, another prophage downstream of tRNA Arg-CCG. In 6179, the third phage is inserted downstream of the tRNA Thr-GGT and in 4423 and 3253 upstream of the tRNA Ser-CGA. Prophages or phage remnants integrated into the $\operatorname{com} K$ gene are found in three of the nine ST121 genomes. Prophages integrated into comK have been suggested to be important for the adaptation of L. monocytogenes to food environments (Verghese et al., 2011).

The phages at tRNA Ser-CGA in 4423 and 3253 and tRNA Thr-CGT in 6179 are specific for the respective strains (but show highest similarity to other Listeria phages). Interestingly, in 6179,4423 , and 3253, the phages at tRNA Arg-CCG are identical and the phages inserted at tRNA Arg-TCT show more than 96.5\% nucleotide sequence identity to each other (Supplementary Figures 1, 2). Highly similar tRNA Arg-CCG (99.8 to $100 \%$ nucleotide identity) and tRNA Arg-TCT (95.4 to 100\% nucleotide identity) prophage contigs are also present in LM_1880, N53-1,

TABLE 2 | General features of the $L$. monocytogenes 6179, 4423, and 3253 genomes and plasmids determined in this study.

\begin{tabular}{|c|c|c|c|c|c|c|}
\hline & 3253 & 4423 & 6179 & pLM3253 & pLM4423 & pLM6179 \\
\hline Assembly size (bp) & $3,001,169^{a}$ & $3,000,849^{a}$ & $3,010,620^{a}$ & $63,152^{\mathrm{a}}$ & 62,207 & 62,206 \\
\hline No. of contigs & 11 & 11 & 1 & 1 & 1 & 1 \\
\hline No. of predicted coding sequences (CDS) & 2990 & 3054 & 3063 & 62 & 62 & 61 \\
\hline Average length of CDS & 890 & 889 & 886 & 892 & 892 & 902 \\
\hline Coding density (\%) & 89.4 & 89.5 & 89.4 & 88.8 & 88.8 & 88.3 \\
\hline No. of tRNA genes & 63 & 61 & 49 & - & - & - \\
\hline No. of prophages & 3 & 3 & 3 & - & - & - \\
\hline
\end{tabular}

${ }^{a}$ Genomes are not closed.

${ }^{b}$ The number of rRNA operons could not be determined due to remaining gaps in the assembly. 


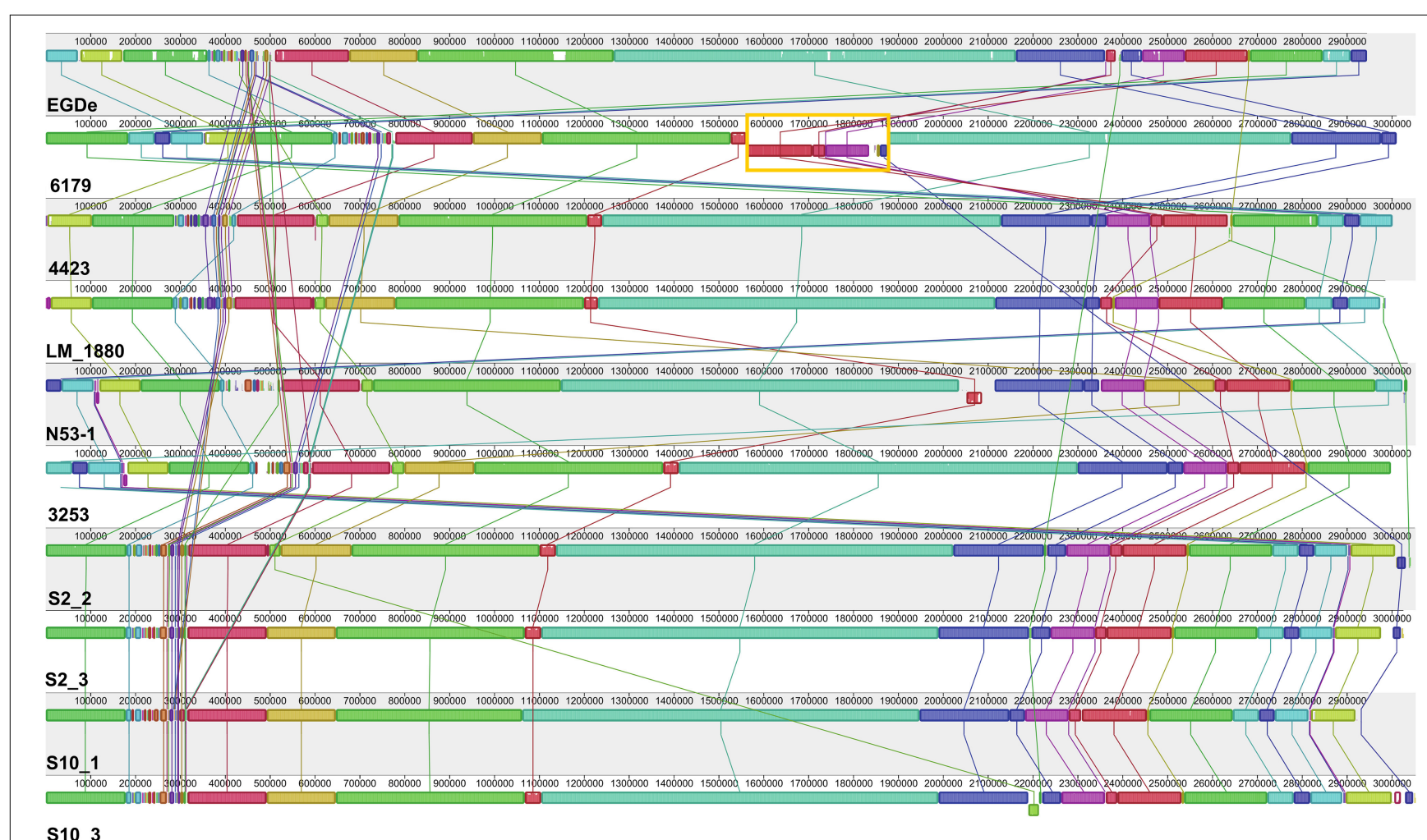

S10_3

FIGURE 1 | Alignment of $L$. monocytogenes ST121 genomes. The genomes of L. monocytogenes EGDe (ST35), 6179, 4423; N53-1, 3253, LM_1880, S2_2, S2_3, S10_1, and S10_3 were aligned using Mauve (Darling et al., 2010). Homologous regions are shown in the same color.
The height of the similarity profile within each block corresponds to the average level of conservation in that region of the genomes. The rearrangement in the $L$. monocytogenes 6179 genome is highlighted in orange.
S2_2, S2_3, S10_1, and S10_3 (Supplementary Figures 1, 2). The similarity of the ST121 tRNA Arg-TCT and tRNA ArgCCG prophages to other described Listeria phages is considerably lower (Supplementary Figures 1,2). This high degree of similarity of prophages-particularly of tRNA-Arg-CCG prophages-in L. monocytogenes ST121 strains isolated independently from food processing plants from five different countries (Austria, Ireland, Denmark, Italy, and Spain) in different years is striking, particularly keeping in mind that prophages have been shown to be important drivers of short-term genome evolution in L. monocytogenes (Orsi et al., 2008; Gilmour et al., 2010; Verghese et al., 2011) and are considered to be the major source of diversity within the genus Listeria (Kuenne et al., 2013). Two recent studies performed comparative genome analyses of Listeria bacteriophages also found regions showing high conservation between some phages, but to a much lesser degree as found in our study (Dorscht et al., 2009; Denes et al., 2014). The high degree of similarity particularly of the tRNA Arg-CCG prophages identified here might thus be a result of adaptation of ST121 strains to similar niches in food production environments. It has been shown that prophages can provide increased growth under nutrient limitation (Edlin et al., 1977), increased biofilm formation (Wang et al., 2010; Verghese et al., 2011; Fortier and Sekulovic, 2013) and can be beneficial for withstanding osmotic, oxidative, and acid stress (Wang et al., 2010). In a similar way, the presence and high conservation of $L$. monocytogenes ST121 prophages might thus be advantageous for survival under stress conditions, which they are faced with in food production environments.

All analyzed ST121 genomes encode a typical L. monocytogenes pathogenicity island and truncated internalin A (inlA) genes, a feature often found in ready-to-eat food and food production environment L. monocytogenes isolates (Nightingale et al., 2008; Van Stelten et al., 2010). The truncated InlA proteins have a predicted length of 492 amino acids and belong to mutation type 6 (Nightingale et al., 2008; Van Stelten et al., 2010). Overall, the genomic organization of the inlAB locus in ST121 strains is similar to L. monocytogenes EGDe (Supplementary Figure 3). Interestingly, the lmo0435 homolog BapL, a putative peptidoglycan bound protein involved in biofilm formation, but not essential (Jordan et al., 2008), is truncated in ST121 strains (Supplementary Figure 3). All ST121 strains encode a highly similar set of internalins and internalin-like proteins (Supplementary Tables 1,2). Using a set of virulence-associated genes based on a study by den Bakker et al. (2010), we performed BlastP and tBlastN searches: all virulence genes present in L. monocytogenes EGDe except homologs of lmo2026 (an internalin-like protein) are present in the ST121 genomes (data not shown). 
Listeria genomes are highly syntenic and horizontal gene transfer into Listeria genomes occurs mostly in the accessory genome consisting mainly of prophages, transposons and so-called hypervariable hotspots (den Bakker et al., 2010, 2013; Kuenne et al., 2013). We thus compared the regions of the hypervariable hotspots in the ST121 genomes to identify possible differences and found the same gene content in all hypervariable hotspots; only in LM_1880, hypervariable hotspot 7 (Imo0458 to $l m o$ 0480) seems to be part of a rearrangement, however also here the gene content is identical to the other ST121 genomes (data not shown). One mechanism for protection against foreign DNA are restriction modification systems. The ST121 genomes encode a lmoJ2 type II restriction modification system locus inserted into hypervariable hotspot 4 (Imo0301 to lmo0314); LmoJ2 has been shown to be involved in phage resistance (Lee et al., 2012). In addition, all ST121 genomes encode two recently described CRISPR loci: a type I CRISPR system inserted between Imo0517 and Imo0518 homologs, and a type II CRISPR system inserted between $l m o 2591$ and $l m o 2595$ as described previously in other L. monocytogenes genomes (Kuenne et al., 2013; Sesto et al., 2014). We analyzed the CRISPR regions in the ST121 genomes: overall, the CRISPR regions were identical, with the exception of a single $14 \mathrm{bp}$ insertion in CRISPR locus 1 in 6179 and the presence of only 21 spacers in 4423 compared to other complete CRISPR 2 loci in ST121 genomes (Supplementary Table 3); however, in 6179, LM_1880, and N53-1 CRISPR 2 loci were located at the end of contigs and thus not completely assembled.

L. monocytogenes encounters various stress conditions in food and food production environments. We therefore analyzed the ST121 genomes with respect to genes possibly involved in stress tolerance. Previously, the so-called stress survival islet 1 (SSI-1) has been characterized in L. monocytogenes and was shown to confer increased tolerance toward acidic and salt stress (Ryan et al., 2010). Similar to the results of a recent study, all ST121 genomes harbor homologs of the L. innocua genes lin0464 and lin0465 at the same genomic locus (Hein et al., 2011). However, their function is currently unknown.

Plasmids also often confer increased stress tolerance. The pLM4423 and pLM6179 plasmid genome sequences were closed by PCR and have a size of $62.2 \mathrm{kbp}$ (Table 2). They encode typical features found in many Listeria plasmids such as determinants for plasmid maintenance, replication and possible transfer as well as the CadAC Cadmium resistance transposon Tn5422 (Lebrun et al., 1994a,b; Kuenne et al., 2010) and also ClpL homologs, which are members of the HSP100 subgroup of heatshock proteins. ClpL proteins were found in some Listeria plasmids before (Kuenne et al., 2010). Highly similar (approximately 68\% amino acid identity) ClpL proteins have been shown to be involved in stress response, virulence under various conditions in Streptococcus spp. and Lactobacillus spp. and to act as chaperone for the stress response regulator CtsR (Suokko et al., 2005; Kajfasz et al., 2009; Tran et al., 2011; Tao and Biswas, 2013). The plasmid-encoded ClpL proteins thus most likely provide additional stress response potential to ST121 strains. Most of the other predicted proteins of ST121 plasmids have no known function. All currently sequenced ST121 L. monocytogenes harbor a plasmid with assembly sizes between 60.9 and $63.1 \mathrm{kbp}$
(Table 1). Based on phylogenetic analyses of plasmid replication initiation proteins, these plasmids belong to group 2 Listeria plasmids (Supplementary Figure 4). Strikingly, these plasmids are basically identical (Figure 2, Supplementary Figure 4) and show more than $99.9 \%$ nucleotide identity to each other. The only visible differences in the alignments (Figure 2) are due to the fact that only the pLM6179 and pLM4423 plasmid sequences are closed. The similarity to their most similar related plasmids is considerably lower: $99.3 \%$ nucleotide identity (coverage: $78 \%$ ) to pLM5578 from L. monocytogenes 08-5578 (ST120) (Gilmour et al., 2010) and $96.6 \%$ (coverage: $74 \%$ ) to pLMR479a from L. monocytogenes R479a (ST8) (Supplementary Figure 5). This extremely high degree of conservation between plasmids of strains isolated from different sources, years and countries suggests that strong selective pressure is acting on the ST121 plasmids. This high conservation of ST121 plasmids may thus be the result of niche adaptation. Plasmids are thought to have important ecological functions because they can be found in high abundance in bacterial populations in diverse habitats and encode a wide array of accessory functions which may confer an advantage to their bacterial hosts, compensating for the burden of carrying a plasmid (Heuer et al., 2008). In addition, it has been suggested that not only bacterial taxa but also their plasmids are defined by their respective ecological niches (Brown Kav et al., 2012). High similarity among some Listeria plasmid sequences has been observed by previous studies (Canchaya et al., 2010; Kuenne et al., 2010). However, in these cases the regions of high similarity represented either only parts of the plasmid sequences or plasmids such as pLM33 with a size of $32 \mathrm{kbp}$ integrated into other larger plasmids and not-as found here-the whole plasmid genomes. Another possible explanation for the high conservation observed in ST121 plasmids and prophages might be that these mobile genetic elements show a high dispersal rate. Assuming a high dispersal rate of these mobile genetic elements nevertheless strongly suggests an advantage of their presence. In line with this, we could show a high level of transcription and differential expression (more than threefold change) of 75 of the tRNA-Arg-TCT and tRNA-Arg-CCG prophage genes and also of 30 out of 62 plasmid genes in L. monocytogenes 6179 under benzethonium chloride challenge (Casey et al., 2014); suggesting an important functional role of these mobile genetic elements at least under the conditions analyzed in this study. In addition, the strong and wide transcription of these genes suggests that negative selection is not acting on these prophages and plasmids.

In food production environments, bacteria are regularly faced with cleaning and disinfectants, thus resistance mechanisms may provide important advantages. Recently, we identified the novel transposon Tn6188 in L. monocytogenes 6179 and 4423, which is involved in increased tolerance to various quaternary ammonium compounds such as benzalkonium chloride. We found Tn6188 in $11 \%$ of the analyzed strains, the Tn6188positive strains were primarily of serovar $1 / 2 \mathrm{a}$ (Muller et al., 2013 , 2014). Interestingly, identical Tn6188 copies are present in all ST121 strains analyzed in this study. However, the Tn6188 copies in N53-1 and LM-1880 were not correctly annotated in these two genomes. In addition, a different benzalkonium chloride resistance mechanism, the bcrABC cassette has been 


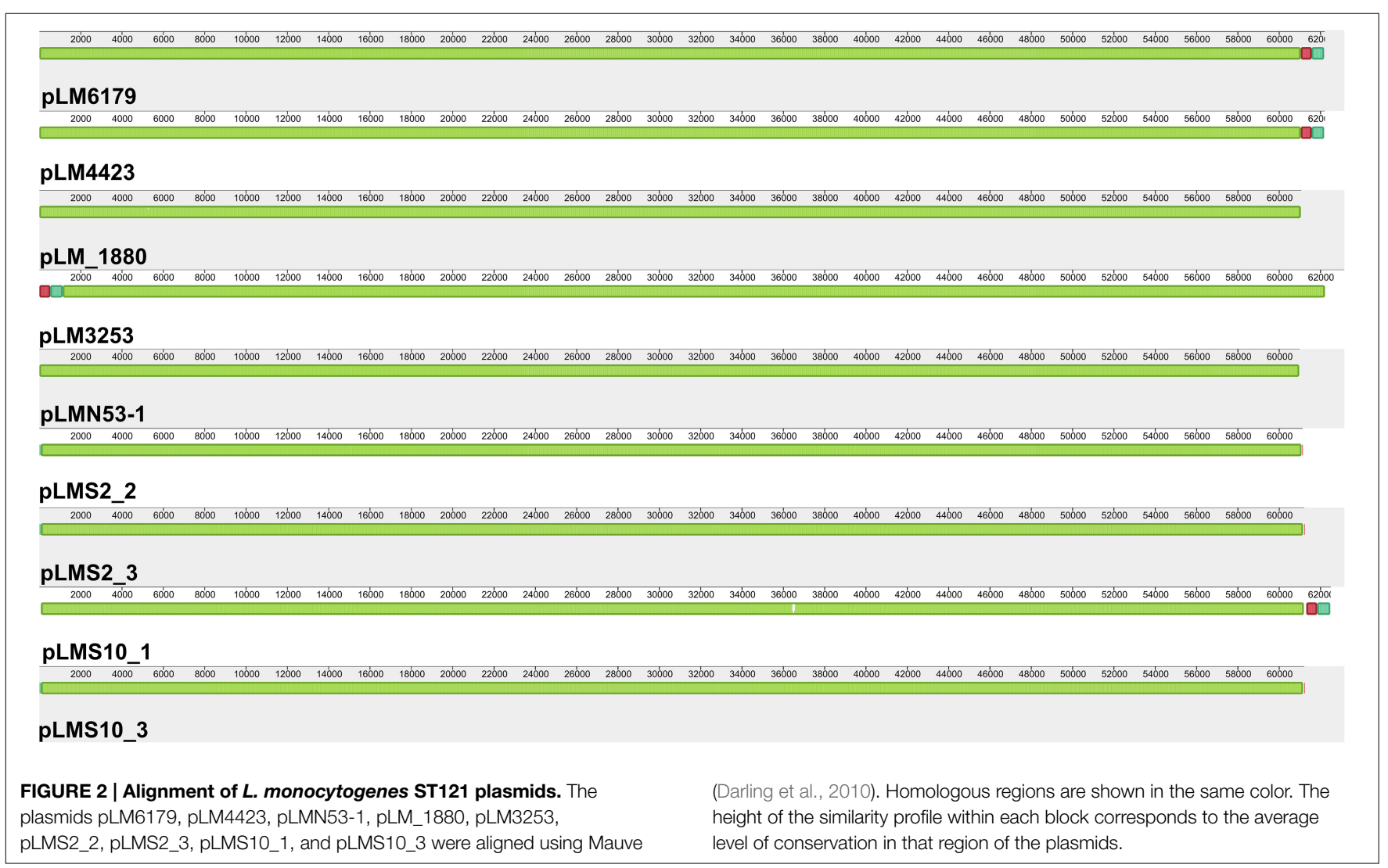

characterized in L. monocytogenes $\mathrm{H} 7550$ (a serovar 4b strain) and was later found in other L. monocytogenes genomes and plasmids (Elhanafi et al., 2010; Dutta et al., 2013). In contrast to Tn6188, bcrABC cassettes are absent from the sequenced ST121 genomes.

We found a $12.5 \mathrm{kbp}$ insertion in the ST121 genomes between the EGDe $1 m o 2753$ and $1 m o 2754$ homologs. This insertion has a $\mathrm{G}+\mathrm{C}$ content of $40.0 \%$, which is slightly higher than the average genomic $\mathrm{G}+\mathrm{C}$ content of $L$. monocytogenes genomes, and encodes-among others-a 3056 amino acid protein harboring 29 rearrangement hotspot (RHS) repeats (PFAM domain: PF05593, locus_tag: LM6179_0173, Figure 3). The RHS proteins and the region surrounding them in ST121 strains are identical. RHS repeat harboring proteins such as RhsAB from E. coli or Dickeya dadantii (the former Erwinia chrysanthemi) or WapA from Bacillus subtilis have been shown to be involved in intercellular competition by inhibiting growth of neighboring cells (Koskiniemi et al., 2013). More generally, RHS proteins have been found in many diverse bacteria and been shown or suggested to be involved in cell-cell interactions (Busby et al., 2013; Kwong et al., 2014). Generally, the conservation among RHS proteins is low (Busby et al., 2013). In line with this, the L. monocytogenes ST 121 RHS proteins show only $25 \%$ amino acid identity to WapA from B. subtilis, which is responsible for tRNA cleavage resulting in inhibition of cell growth of neighboring cells (Koskiniemi et al., 2013). Upstream of the RHS protein in ST121 strains, a putative RNA $2^{\prime}$-phosphotransferase (KptA) is present (locus_tag: LM6179_0169). The ST121 KptA proteins show $39 \%$ amino acid identity to the functionally characterized E. coli KptA. Interestingly, RNA $2^{\prime}$-phopsphotransferases have been shown to perform RNA cleavage by a mechanism highly similar to ADP-ribosylation catalyzed by various bacterial toxins (Spinelli et al., 1999). Although not directly shown, most likely, KptA also catalyzes tRNA cleavage (Spinelli et al., 1999). Thus, the putative RNA 2'-phosphotransferases in ST121 strains might fulfill a similar function than the nuclease domain of WapA in B. subtilis. In this context it is tempting to speculate that the presence of this insertion might relate to the widespread occurrence of L. monocytogenes ST121 strains in food production environments. We hypothesize that the presence of this insertion harboring the RHS protein and the putative RNA $2^{\prime}$-phosphotransferases provides ST121 strains with a means for better competition against other bacteria in food production environments.

Taken together, our results show that L. monocytogenes ST121 strains are highly similar to each other and also show an extremely high degree of conservation in some prophage regions and in their plasmids. This high level of conservation suggests that a strong selective pressure is acting on these conserved prophages and plasmids, which are usually among the most variable parts of genetic information in bacteria. It is tempting to speculate that these conserved regions provide ST121 strains with fitness adaptations possibly enhancing survival in food production environments as shown e.g., for other prophages. In 


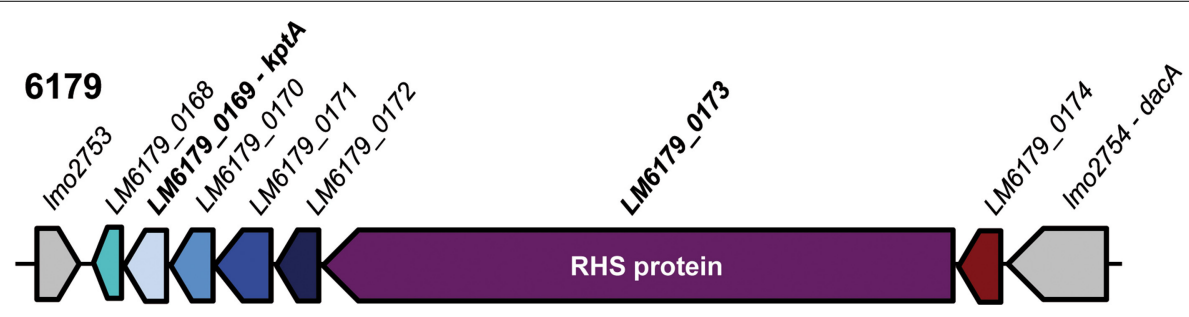

4423

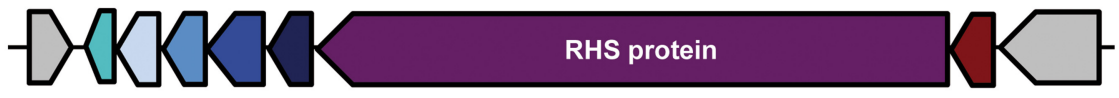

N53-1

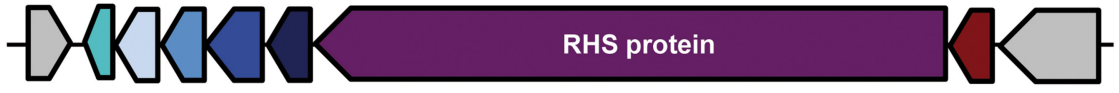

LM_1880

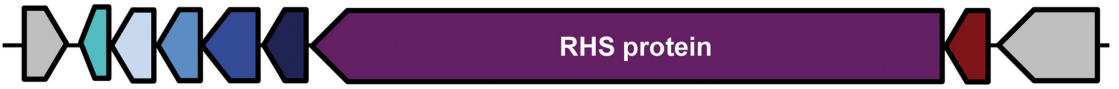

3253

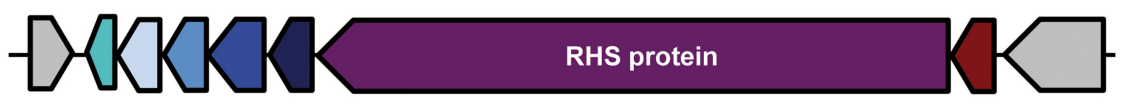

S2_2

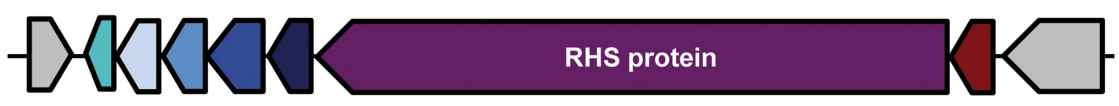

FIGURE 3 | Organization of the insertion between Imo2753 and Imo2754 homologs in L. monocytogenes ST121 strains. Homologous genes are shown in the same color. The inserted region is identical in all $\overline{1 \mathrm{kbp}}$

analyzed ST121 genomes. For clarity, only one of the Spanish pork industry isolates (S2_2) and only L. monocytogenes 6179 locus_tags are shown.

\section{Acknowledgments}

common genetic determinants such as Tn6188, or the insertion harboring the RHS proteins, which might also increase their chances of becoming persistent. However, more L. monocytogenes ST121 genome sequences, genomes from persistent strains from other sequence types and experimental validation will be needed to confirm our hypotheses. A possible contribution of L. monocytogenes ST121 plasmids to survival in food production environments could, e.g., be tested by curing strains from their plasmids or by deleting specific plasmid genes of interest such as the genes encoding the ClpL proteins. Similarly, a contribution of prophages to persistence could be tested by deleting the prophages and monitoring the phenotypic effects on survival under conditions similar to food production environments. Likewise, a possible role of other chromosomally encoded genes in persistence such as the RHS proteins could be analyzed by generating deletion mutants and investigating their effect on survival.
Christian Schlötterer and Viola Nolte are acknowledged for genome sequencing and assembly of L. monocytogenes strain 6179 , which was kindly provided by Kieran Jordan. MW and SS are members of the EU COST Action FA1202 (CGA-FA1202): "A European Network for Mitigating Bacterial Colonisation and Persistence on Foods and Food Processing Environments" (http://www.bacfoodnet.org/) and acknowledge this action for facilitating collaborative networking that assisted with this study. This study was supported by the EU 7th Framework Programme project PROMISE (project number 265877).

\section{Supplementary Material}

The Supplementary Material for this article can be found online at: http://journal.frontiersin.org/article/10.3389/fmicb. 2015.00380/abstract 


\section{References}

Allerberger, F., and Wagner, M. (2010). Listeriosis: a resurgent foodborne infection. Clin. Microbiol. Infect. 16, 16-23. doi: 10.1111/j.1469-0691.2009.03109.x

Althaus, D., Lehner, A., Brisse, S., Maury, M., Tasara, T., and Stephan, R. (2014). Characterization of Listeria monocytogenes strains isolated during 2011-2013 from human infections in Switzerland. Foodborne Pathog. Dis. 11, 753-758. doi: 10.1089/fpd.2014.1747

Aziz, R. K., Bartels, D., Best, A. A., Dejongh, M., Disz, T., Edwards, R. A., et al. (2008). The RAST server: rapid annotations using subsystems technology. BMC Genomics 9:75. doi: 10.1186/1471-2164-9-75

Brown Kav, A., Sasson, G., Jami, E., Doron-Faigenboim, A., Benhar, I., and Mizrahi, I. (2012). Insights into the bovine rumen plasmidome. Proc. Natl. Acad. Sci. U.S.A. 109, 5452-5457. doi: 10.1073/pnas.1116410109

Busby, J. N., Panjikar, S., Landsberg, M. J., Hurst, M. R., and Lott, J. S. (2013). The BC component of $\mathrm{ABC}$ toxins is an RHS-repeat-containing protein encapsulation device. Nature 501, 547-550. doi: 10.1038/nature 12465

Camacho, C., Coulouris, G., Avagyan, V., Ma, N., Papadopoulos, J., Bealer, K., et al. (2009). BLAST+: architecture and applications. BMC Bioinformatics 10:421. doi: 10.1186/1471-2105-10-421

Canchaya, C., Giubellini, V., Ventura, M., de los Reyes-Gavilan, C. G., and Margolles, A. (2010). Mosaic-like sequences containing transposon, phage, and plasmid elements among Listeria monocytogenes plasmids. Appl. Environ. Microbiol. 76, 4851-4857. doi: 10.1128/AEM.02799-09

Carpentier, B., and Cerf, O. (2011). Review-persistence of Listeria monocytogenes in food industry equipment and premises. Int. J. Food Microbiol. 145, 1-8. doi: 10.1016/j.ijfoodmicro.2011.01.005

Casey, A., Fox, E. M., Schmitz-Esser, S., Coffey, A., McAuliffe, O., and Jordan, K. (2014). Transcriptome analysis of Listeria monocytogenes exposed to biocide stress reveals a multi-system response involving cell wall synthesis, sugar uptake, and motility. Front. Microbiol. 5:68. doi: 10.3389/fmicb.2014.00068

Chenal-Francisque, V., Lopez, J., Cantinelli, T., Caro, V., Tran, C., Leclercq, A., et al. (2011). Worldwide distribution of major clones of Listeria monocytogenes. Emerging Infect. Dis. 17, 1110-1112. doi: 10.3201/eid/1706.101778

Chiara, M., D'erchia, A. M., Manzari, C., Minotto, A., Montagna, C., Addante, N., et al. (2014). Draft genome sequences of six Listeria monocytogenes strains isolated from dairy products from a processing plant in southern Italy. Genome Announc. 2:e00282-14. doi: 10.1128/genomeA.00282-14

Darling, A. E., Mau, B., and Perna, N. T. (2010). progressiveMauve: multiple genome alignment with gene gain, loss and rearrangement. PLoS ONE 5:e11147. doi: 10.1371/journal.pone.0011147

den Bakker, H. C., Cummings, C. A., Ferreira, V., Vatta, P., Orsi, R. H., Degoricija, L., et al. (2010). Comparative genomics of the bacterial genus Listeria: genome evolution is characterized by limited gene acquisition and limited gene loss. BMC Genomics 11:688. doi: 10.1186/1471-2164-11-688

den Bakker, H. C., Desjardins, C. A., Griggs, A. D., Peters, J. E., Zeng, Q., Young, S. K., et al. (2013). Evolutionary dynamics of the accessory genome of Listeria monocytogenes. PLoS ONE 8:e67511. doi: 10.1371/journal.pone.0067511

Denes, T., Vongkamjan, K., Ackermann, H. W., Moreno Switt, A. I., Wiedmann, M., and den Bakker, H. C. (2014). Comparative genomic and morphological analyses of Listeria phages isolated from farm environments. Appl. Environ. Microbiol. 80, 4616-4625. doi: 10.1128/AEM.00720-14

Dorscht, J., Klumpp, J., Bielmann, R., Schmelcher, M., Born, Y., Zimmer, M., et al. (2009). Comparative genome analysis of Listeria bacteriophages reveals extensive mosaicism, programmed translational frameshifting, and a novel prophage insertion site. J. Bacteriol. 191, 7206-7215. doi: 10.1128/JB.01041-09

Dutta, V., Elhanafi, D., and Kathariou, S. (2013). Conservation and distribution of the benzalkonium chloride resistance cassette bcrABC in Listeria monocytogenes. Appl. Environ. Microbiol. 79, 6067-6074. doi: 10.1128/AEM.01751-13

Edlin, G., Lin, L., and Bitner, R. (1977). Reproductive fitness of P1, P2, and Mu lysogens of Escherichia coli. J. Virol. 21, 560-564.

Elhanafi, D., Dutta, V., and Kathariou, S. (2010). Genetic characterization of plasmid-associated benzalkonium chloride resistance determinants in a Listeria monocytogenes strain from the 1998-1999 outbreak. Appl. Environ. Microbiol. 76, 8231-8238. doi: 10.1128/AEM.02056-10
Eurosurveillance Editorial, T. (2012). The European Union summary report on trends and sources of zoonoses, zoonotic agents and foodborne outbreaks in 2010. Euro Surveill. 17:2597. doi: 10.2903/j.efsa. 2012.2597

Ferreira, V., Wiedmann, M., Teixeira, P., and Stasiewicz, M. J. (2014). Listeria monocytogenes persistence in food-associated environments: epidemiology, strain characteristics, and implications for public health. J. Food Prot. 77, 150-170. doi: 10.4315/0362-028X.JFP-13-150

Fortier, L. C., and Sekulovic, O. (2013). Importance of prophages to evolution and virulence of bacterial pathogens. Virulence 4, 354-365. doi: 10.4161/viru.24498

Fox, E., Hunt, K., O'brien, M., and Jordan, K. (2011a). Listeria monocytogenes in Irish farmhouse cheese processing environments. Int. J. Food Microbiol. 145(Suppl. 1), S39-S45. doi: 10.1016/j.ijfoodmicro.2010.10.012

Fox, E. M., Leonard, N., and Jordan, K. (2011b). Physiological and transcriptional characterization of persistent and nonpersistent Listeria monocytogenes isolates. Appl. Environ. Microbiol. 77, 6559-6569. doi: 10.1128/AEM.05529-11

Gilmour, M., Graham, M., Van Domselaar, G., Tyler, S., Kent, H., TroutYakel, K., et al. (2010). High-throughput genome sequencing of two Listeria monocytogenes clinical isolates during a large foodborne outbreak. $B M C$ Genomics 11:120. doi: 10.1186/1471-2164-11-120

Grissa, I., Vergnaud, G., and Pourcel, C. (2007). CRISPRFinder: a web tool to identify clustered regularly interspaced short palindromic repeats. Nucleic Acids Res. 35, W52-W57. doi: 10.1093/nar/gkm360

Hein, I., Klinger, S., Dooms, M., Flekna, G., Stessl, B., Leclercq, A., et al. (2011). Stress Survival Islet 1 (SSI-1) survey in Listeria monocytogenes reveals an insert common to Listeria innocua in sequence type 121 L. monocytogenes strains. Appl. Environ. Microbiol. 77, 2169-2173. doi: 10.1128/AEM.02159-10

Heuer, H., Abdo, Z., and Smalla, K. (2008). Patchy distribution of flexible genetic elements in bacterial populations mediates robustness to environmental uncertainty. FEMS Microbiol. Ecol. 65, 361-371. doi: 10.1111/j.15746941.2008.00539.x

Holch, A., Webb, K., Lukjancenko, O., Ussery, D., Rosenthal, B. M., and Gram, L. (2013). Genome sequencing identifies two nearly unchanged strains of persistent Listeria monocytogenes isolated at two different fish processing plants sampled 6 years apart. Appl. Environ. Microbiol. 79, 2944-2951. doi: 10.1128/AEM.03715-12

Jordan, S. J., Perni, S., Glenn, S., Fernandes, I., Barbosa, M., Sol, M., et al. (2008). Listeria monocytogenes biofilm-associated protein (BapL) may contribute to surface attachment of $L$. monocytogenes but is absent from many field isolates. Appl. Environ. Microbiol. 74, 5451-5456. doi: 10.1128/AEM.02419-07

Kaifasz, J. K., Martinez, A. R., Rivera-Ramos, I., Abranches, J., Koo, H., Quivey, R. G. Jr., et al. (2009). Role of Clp proteins in expression of virulence properties of Streptococcus mutans. J. Bacteriol. 191, 2060-2068. doi: 10.1128/JB. 01609-08

Kastbjerg, V. G., Hein-Kristensen, L., and Gram, L. (2014). Triclosaninduced aminoglycoside-tolerant Listeria monocytogenes isolates can appear as small-colony variants. Antimicrob. Agents Chemother. 58, 3124-3132. doi: 10.1128/AAC.02266-13

Koskiniemi, S., Lamoureux, J. G., Nikolakakis, K. C., T'kint de Roodenbeke, C., Kaplan, M. D., Low, D. A., et al. (2013). RHS proteins from diverse bacteria mediate intercellular competition. Proc. Natl. Acad. Sci. U.S.A. 110, 7032-7037. doi: 10.1073/pnas.1300627110

Kuenne, C., Billion, A., Mraheil, M. A., Strittmatter, A., Daniel, R., Goesmann, A., et al. (2013). Reassessment of the Listeria monocytogenes pan-genome reveals dynamic integration hotspots and mobile genetic elements as major components of the accessory genome. BMC Genomics 14:47. doi: 10.1186/14712164-14-47

Kuenne, C., Voget, S., Pischimarov, J., Oehm, S., Goesmann, A., Daniel, R., et al. (2010). Comparative analysis of plasmids in the genus Listeria. PLoS ONE 5:e12511. doi: 10.1371/journal.pone.0012511

Kwong, W. K., Engel, P., Koch, H., and Moran, N. A. (2014). Genomics and host specialization of honey bee and bumble bee gut symbionts. Proc. Natl. Acad. Sci. U.S.A. 111, 11509-11514. doi: 10.1073/pnas.1405838111

Larsen, M. H., Dalmasso, M., Ingmer, H., Langsrud, S., Malakauskas, M., Mader, A., et al. (2014). Persistence of foodborne pathogens and their control in primary and secondary food production chains. Food Control 44, 92-109. doi: 10.1016/j.foodcont.2014.03.039 
Larsen, M. V., Cosentino, S., Rasmussen, S., Friis, C., Hasman, H., Marvig, R. L., et al. (2012). Multilocus sequence typing of total-genome-sequenced bacteria. J. Clin. Microbiol. 50, 1355-1361. doi: 10.1128/JCM.06094-11

Lebrun, M., Audurier, A., and Cossart, P. (1994a). Plasmid-borne cadmium resistance genes in Listeria monocytogenes are present on $\operatorname{Tn} 5422$, a novel transposon closely related to Tn917. J. Bacteriol. 176, 3049-3061.

Lebrun, M., Audurier, A., and Cossart, P. (1994b). Plasmid-borne cadmium resistance genes in Listeria monocytogenes are similar to cadA and cadC of Staphylococcus aureus and are induced by cadmium. J. Bacteriol. 176, 3040-3048.

Lee, S., Ward, T. J., Siletzky, R. M., and Kathariou, S. (2012). Two novel type II restriction-modification systems occupying genomically equivalent locations on the chromosomes of Listeria monocytogenes strains. Appl. Environ. Microbiol. 78, 2623-2630. doi: 10.1128/AEM.07203-11

Lopez-Alonso, V., Ortiz, S., and Martinez-Suarez, J. V. (2015). Genome sequences of five disinfectant-resistant Listeria monocytogenes strains from two Iberian pork-processing plants. Genome Announc. 3:e00077-15. doi: 10.1128/genomeA.00077-15

Martin, B., Perich, A., Gomez, D., Yanguela, J., Rodriguez, A., Garriga, M., et al. (2014). Diversity and distribution of Listeria monocytogenes in meat processing plants. Food Microbiol. 44, 119-127. doi: 10.1016/j.fm.2014.05.014

Muller, A., Rychli, K., Muhterem-Uyar, M., Zaiser, A., Stessl, B., Guinane, C. M., et al. (2013). Tn6188-a novel transposon in Listeria monocytogenes responsible for tolerance to benzalkonium chloride. PLOS ONE 8:e76835. doi: 10.1371/journal.pone. 0076835

Muller, A., Rychli, K., Zaiser, A., Wieser, C., Wagner, M., and Schmitz-Esser, S. (2014). The Listeria monocytogenes transposon Tn6188 provides increased tolerance to various quaternary ammonium compounds and ethidium bromide. FEMS Microbiol. Lett. 361, 166-173. doi: 10.1111/1574-6968.12626

Nightingale, K. K., Ivy, R. A., Ho, A. J., Fortes, E. D., Njaa, B. L., Peters, R. M., et al. (2008). inlA premature stop codons are common among Listeria monocytogenes isolates from foods and yield virulence-attenuated strains that confer protection against fully virulent strains. Appl. Environ. Microbiol. 74, 6570-6583. doi: 10.1128/AEM.00997-08

Orsi, R. H., Borowsky, M. L., Lauer, P., Young, S. K., Nusbaum, C., Galagan, J. E., et al. (2008). Short-term genome evolution of Listeria monocytogenes in a noncontrolled environment. BMC Genomics 9:539. doi: 10.1186/1471-2164-9-539

Ortiz, S., Lopez, V., and Martinez-Suarez, J. V. (2014). Control of Listeria monocytogenes contamination in an Iberian pork processing plant and selection of benzalkonium chloride-resistant strains. Food Microbiol. 39, 81-88. doi: 10.1016/j.fm.2013.11.007

Ortiz, S., Lopez, V., Villatoro, D., Lopez, P., Davila, J. C., and Martinez-Suarez, J. N. (2010). A 3-year surveillance of the genetic diversity and persistence of Listeria monocytogenes in an Iberian pig slaughterhouse and processing plant. Foodborne Pathog. Dis. 7, 1177-1184. doi: 10.1089/fpd.2010.0535

Overbeek, R., Olson, R., Pusch, G. D., Olsen, G. J., Davis, J. J., Disz, T., et al. (2014). The SEED and the rapid annotation of microbial genomes using subsystems technology (RAST). Nucleic Acids Res. 42, D206-D214. doi: 10.1093/nar/gkt1226

Parisi, A., Latorre, L., Normanno, G., Miccolupo, A., Fraccalvieri, R., Lorusso, V., et al. (2010). Amplified fragment length polymorphism and multi-locus sequence typing for high-resolution genotyping of Listeria monocytogenes from foods and the environment. Food Microbiol. 27, 101-108. doi: 10.1016/j.fm.2009.09.001

Ragon, M., Wirth, T., Hollandt, F., Lavenir, R., Lecuit, M., Le Monnier, A., et al. (2008). A new perspective on Listeria monocytogenes evolution. PLoS Pathog. 4:e1000146. doi: 10.1371/journal.ppat.1000146

Ryan, S., Begley, M., Hill, C., and Gahan, C. G. (2010). A five-gene stress survival islet (SSI-1) that contributes to the growth of Listeria monocytogenes in suboptimal conditions. J. Appl. Microbiol. 109, 984-995. doi: 10.1111/j.13652672.2010.04726.x
Sauders, B. D., and Wiedmann, M. (2007). "Ecology of Listeria species and L. monocytogenes in the natural environment," in Listeria, Listeriosis and Food Safety, 3rd Edn., eds E. T. Ryser and E. H. Marth (Boca Raton, FL: CRC Press), $21-53$.

Sesto, N., Touchon, M., Andrade, J. M., Kondo, J., Rocha, E. P., Arraiano, C. M., et al. (2014). A PNPase dependent CRISPR System in Listeria. PLoS Genet. 10:e1004065. doi: 10.1371/journal.pgen.1004065

Spinelli, S. L., Kierzek, R., Turner, D. H., and Phizicky, E. M. (1999). Transient ADP-ribosylation of a $2^{\prime}$-phosphate implicated in its removal from ligated tRNA during splicing in yeast. J. Biol. Chem. 274, 2637-2644. doi: $10.1074 / \mathrm{jbc} .274 .5 .2637$

Stessl, B., Fricker, M., Fox, E., Karpiskova, R., Demnerova, K., Jordan, K., et al. (2014). Collaborative survey on the colonization of different types of cheeseprocessing facilities with Listeria monocytogenes. Foodborne Pathog. Dis. 11, 8-14. doi: 10.1089/fpd.2013.1578

Suokko, A., Savijoki, K., Malinen, E., Palva, A., and Varmanen, P. (2005). Characterization of a mobile clpL gene from Lactobacillus rhamnosus. Appl. Environ. Microbiol. 71, 2061-2069. doi: 10.1128/AEM.71.4.2061-2069.2005

Tamura, K., Stecher, G., Peterson, D., Filipski, A., and Kumar, S. (2013). MEGA6: molecular evolutionary genetics analysis version 6.0. Mol. Biol. Evol. 30, 2725-2729. doi: 10.1093/molbev/mst197

Tao, L., and Biswas, I. (2013). ClpL is required for folding of CtsR in Streptococcus mutans. J. Bacteriol. 195, 576-584. doi: 10.1128/JB.01743-12

Tran, T. D., Kwon, H. Y., Kim, E. H., Kim, K. W., Briles, D. E., Pyo, S., et al. (2011). Decrease in penicillin susceptibility due to heat shock protein ClpL in Streptococcus pneumoniae. Antimicrob. Agents Chemother. 55, 2714-2728. doi: 10.1128/AAC.01383-10

Vallenet, D., Belda, E., Calteau, A., Cruveiller, S., Engelen, S., Lajus, A., et al. (2013). MicroScope-an integrated microbial resource for the curation and comparative analysis of genomic and metabolic data. Nucleic Acids Res. 41, D636-D647. doi: 10.1093/nar/gks1194

Van Stelten, A., Simpson, J. M., Ward, T. J., and Nightingale, K. K. (2010). Revelation by single-nucleotide polymorphism genotyping that mutations leading to a premature stop codon in inlA are common among Listeria monocytogenes isolates from ready-to-eat foods but not human listeriosis cases. Appl. Environ. Microbiol. 76, 2783-2790. doi: 10.1128/AEM. 02651-09

Verghese, B., Lok, M., Wen, J., Alessandria, V., Chen, Y., Kathariou, S., et al. (2011). comK prophage junction fragments as markers for Listeria monocytogenes genotypes unique to individual meat and poultry processing plants and a model for rapid niche-specific adaptation, biofilm formation, and persistence. Appl. Environ. Microbiol. 77, 3279-3292. doi: 10.1128/AEM. 00546-11

Wang, G. Y., Qian, W. J., Zhang, X. X., Wang, H. H., Ye, K. P., Bai, Y., et al. (2015). Prevalence, genetic diversity and antimicrobial resistance of Listeria monocytogenes isolated from ready-to-eat meat products in Nanjing, China. Food Control 50, 202-208. doi: 10.1016/j.foodcont.2014.07.057

Wang, X., Kim, Y., Ma, Q., Hong, S. H., Pokusaeva, K., Sturino, J. M., et al. (2010). Cryptic prophages help bacteria cope with adverse environments. Nat. Commun. 1:147. doi: 10.1038/ncomms1146

Conflict of Interest Statement: The authors declare that the research was conducted in the absence of any commercial or financial relationships that could be construed as a potential conflict of interest.

Copyright $\odot 2015$ Schmitz-Esser, Müller, Stessl and Wagner. This is an open-access article distributed under the terms of the Creative Commons Attribution License (CC $B Y$ ). The use, distribution or reproduction in other forums is permitted, provided the original author(s) or licensor are credited and that the original publication in this journal is cited, in accordance with accepted academic practice. No use, distribution or reproduction is permitted which does not comply with these terms. 